GRADIATION\&APPLICATIONS

ISSN 2466-4294 (online) | rad-journal.org

Vol. 2 | Issue 2 | pp. 118 - 123, 2017

doi: 10.21175/RadJ.2017.02.025

Original research paper

\title{
CHARACTERISTICS OF 1.6 MeV PROTON-IRRADIATED GaN-BASED SENSORS*
}

\author{
Dovile Meskauskaite ${ }^{* *}$, Eugenijus Gaubas, Tomas Ceponis, \\ Jevgenij Pavlov, Vytautas Rumbauskas
}

\author{
Vilnius University, Institute of Applied Research, Vilnius, Lithuania
}

\begin{abstract}
High response speed sensors made of thin GaN-based structures can be important for the optical readout of the radiation signals in harsh radiation environment at hadron accelerator facilities. In this work, the metalsemiconductor-metal structure sensors formed on the MOCVD grown GaN heterostructures have been studied. The proton-induced luminescence (PI-L) and the BELIV (barrier evaluation by linearly increasing voltage) transients have simultaneously been recorded during $1.6 \mathrm{MeV}$ proton irradiation emitted by a Tandetron type accelerator. The $P I-L$ and BELIV measurements allowed for tracing the evolution of the parameters of recombination. The radiation damage on GaN-based sensors has been examined by capacitance-voltage $(C-V)$ and deep-level transient spectroscopy (DLTS) methods. The dominant radiation defects introduced by $1.6 \mathrm{MeV}$ proton beam have been unveiled.
\end{abstract}

Key words: MOCVD GaN, proton irradiation, radiation defects

\section{INTRODUCTION}

GaN offers many advantages over the silicon material for sensor fabrication due to the higher breakdown field, lower leakage currents and elevated operating temperatures [1]. These properties make GaN a promising material for applications in chemical, gas, biological and pressure sensors [2], radiation imaging systems [3] and high energy particle detectors [4]-[5]. GaN can be used for detection and tracking of high-energy particles or dosimetry by recording both the electrical and optical signals [6]-[7].

In this work, the multi-quantum-well-(MQW)based GaN structures grown by MOCVD (metalorganic chemical vapour deposition) technology were investigated in order to trace the evolution of radiation damage. The proton-induced luminescence (PI-L) and the BELIV (barrier evaluation by linearly increasing voltage) transients were simultaneously recorded during exposure to the $1.6 \mathrm{MeV}$ proton beam. The dominant radiation defects introduced by $1.6 \mathrm{MeV}$ proton beam have also been ex situ examined by the deep-level transient spectroscopy (DLTS).

\section{SAMPLES AND EXPERIMENTAL SETUPS}

The $1 \mu \mathrm{m}$ thick InGaN/GaN metal-heterostructuremetal sensors made of commercial light emitting diodes (LEDs, Optosupply OSB4XNE1E1E [8]) were investigated. The multilayer heterostructures of 0.25 $\mu \mathrm{m}$ thickness and $1 \times 10^{-2} \mathrm{~cm}^{2}$ area were grown on sapphire substrates by MOCVD technology. This LED structure also contains a multi-quantum-well (MQW) layer. These sensors contained contacts (100 nm thick) to a sandwich structure.

Several techniques were applied to have the baseline characteristics before irradiations. Temperature-dependent BELIV [9] measurements were performed to identify the barrier capacitance, to separate the current components attributed to the carrier extraction as well as generation and to evaluate carrier traps in device material. The effective doping concentration of $N_{\text {eff }}=3.5 \times 10^{17} \mathrm{~cm}^{-3}$ has been evaluated at room temperature for a pristine sensor, by using BELIV technique. The proton-induced luminescence (PI-L) spectra and the BELIV transients have simultaneously been recorded during $1.6 \mathrm{MeV}$ proton irradiation emitted by a linear particle accelerator Tandetron 4110A. There, the $17 \mu \mathrm{m}$ stopping range for 1.6 MeV protons in GaN was estimated by using the Monte Carlo code SRIM (Stopping and Range of Ions in Matter) [10]. Therefore, $1.6 \mathrm{MeV}$ protons are penetrative for the $1 \mu \mathrm{m}$ thick $\mathrm{InGaN} / \mathrm{GaN}$ heterostucture sensors.

For in situ measurements, the GaN-based sensors were installed within a proprietary vacuum chamber [11]. The coaxial and fiber-optics probes allow for simultaneous recording of the electrical (BELIV, $I_{S C}$ ) and optical (PI-L) signals. The BELIV circuitry contains an adjusted output of a generator of the

\footnotetext{
* The paper was presented at the Fifth International Conference on Radiation and Applications in Various Fields of Research (RAD 2017), Budva, Montenegro, 2017.

dovile.meskauskaite@tmi.vu.lt
} 
D. Meskauskaite et al., Characteristics of 1.6 MeV proton-irradiated..., Rad. Applic., 2017, 2, 2, 118-123

linearly increasing voltage (LIV), a sensor under test and a load resistor $\left(\mathrm{R}_{\mathrm{L}}\right)$, connected in series. The BELIV signal is recorded by a DSO6102A oscilloscope using a $50 \Omega$ load input. The proton induced luminescence spectra were recorded using an Avantes AvaSpec 2048 TEC spectrometer.

The deep level transient spectroscopy (DLTS) technique was ex situ applied to identify the deep traps in the irradiated samples. The capacitance-DLTS (CDLTS) technique is used to trace the time-varied barrier capacitance transients dependent on temperature, determined by the carrier thermal emission from deep levels [12]. The DLTS technique served for the evaluation of the thermal activation energy, of capture cross-sections for electrons and holes, and for estimation of the concentration of carrier traps as well as the trap distribution within structure depth [12]. C-DLTS spectra over temperature range of $10-400 \mathrm{~K}$ were recorded by using a HERA-DLTS System 1030 spectrometer [13]. The PhysTech software installed within HERA-DLTS spectrometer was employed for analysing of the measured DLTS spectra.

\section{RESULTS AND DISCUSSION}

\subsection{BELIV characteristics of pristine sensors}

The BELIV transients, recorded using the pulse technique for barrier evaluation by linearly increasing voltage (LIV), was applied as a fast tool for the characterization of the barrier capacitance, for separation of the current components ascribed to carrier extraction as well as generation and for the estimation of carrier traps within a device material. The BELIV transients were examined varying temperature from 25 to $70{ }^{\circ} \mathrm{C}$ and using reverse $\left(U_{R}\right)$ polarity LIV pulses. There, BELIV transients were recorded using fixed reverse $\left(U_{R}\right)$ voltage pulses with a peak value of $U_{P}=8 \mathrm{~V}$ and pulse duration of $\tau_{P}=50 \mu \mathrm{s}$.

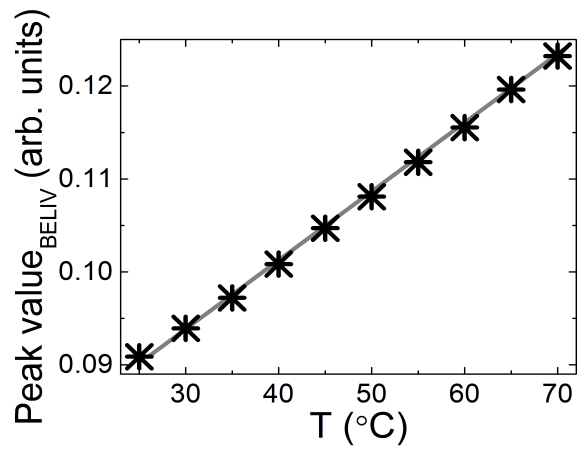

Figure 1. The barrier capacitance (peak value) dependence on temperature

The initial current peak within a BELIV transient, which appeared due to barrier capacitance charging, corresponds to the barrier capacitance value $C_{b o}$, which is dependent on the effective doping concentration $\sim N_{\text {eff }} / / 2$ [14]. As shown in Fig. 1, the amplitude of this peak increases linearly with enhancement of temperature. This result implies the increase of the effective doping $N_{e f f}=N_{D} \pm N_{T}$ due to trap ionization, where $N_{D}$ is the donor concentration and $N_{T}$ is the concentration of the net space charge produced by ionized traps, as these could be positively or negatively charged defects [15]. The effective doping concentration, as determined from BELIV measurements at room temperature, is $N_{\text {eff }}=3.5 \times 10^{17} \mathrm{~cm}^{-3}$.

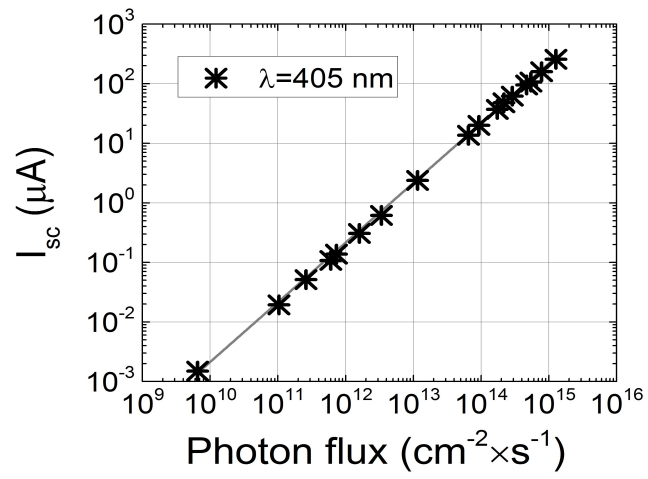

Figure 2. The violet-light flux dependent photoresponse (short-circuit current) recorded on the pristine sensor

The short-circuit current $\left(I_{S C}\right)$ dependence on photon flux $(F)$ of the violet-light illumination $(\lambda=405$ $\mathrm{nm}$ ) is illustrated in Fig.2, as obtained for a nonirradiated sensor. The flux of the light source was varied using optical filters and calibrated using a ThorLabs power-meter PM10oD with S120VC, while a beam cross-sectional area was controlled by using a diaphragm. The obtained near-linear $I_{S C}-F$ dependence (within a $\log -\log$ scale) implies suitability of $\mathrm{GaN}$ based structures for the detection of irradiation characteristics.

\subsection{Variations of characteristics during proton irradiation}

Tracing of evolution of the radiation defects during irradiation can be a dosimetry tool based on the GaNsensors. Owing to the GaN sensor availability to record both the electrical and optical signals, the impact of the radiation defects introduced by a proton beam was studied at room temperature by combining the in situ measurements of the BELIV and proton-induced luminescence (PI-L) characteristics.

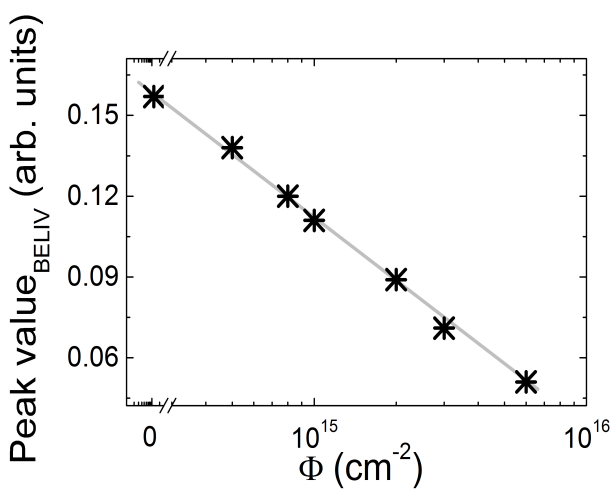

Figure 3. Fluence-dependent barrier charging current measured during irradiation at room temperature 
D. Meskauskaite et al., Characteristics of $1.6 \mathrm{MeV}$ proton-irradiated ..., Rad. Applic., 2017, 2, 2, 118-123

The fluence-dependent peak values of the BELIV current (Fig. 3) indicate the decrease of the barrier capacitance $C_{b o}$ with enhancement of irradiation fluence. This result indicates that radiation-induced defects partially compensate the dopants introduced by LED fabrication. At the highest irradiation fluence $\left(\Phi=6 \times 10^{15} \mathrm{~cm}^{-2}\right)$, the BELIV signal decreases by a factor of 3 , compared to its value in the pristine sensor. The BELIV signal in sensor irradiated with the highest fluence acquires a square-wave shape, which is typical for a fully-depleted sensor where geometrical capacitance determines the BELIV transient. This result implies that the heterojunction sensor under heavy irradiation approaches to the capacitor-like device. The observed decrease of the peak values of BELIV current indicates the changes of the effective doping concentration from $N_{e f f}=3.5 \times 10^{17} \mathrm{~cm}^{-3}$, for a pristine sensor, to $N_{\text {eff }}=2.4 \times 10^{17} \mathrm{~cm}^{-3}$, for the sensor irradiated with the highest fluence. The depletion width of $\sim 250 \mathrm{~nm}$ has been estimated for the sensor irradiated with the proton fluence of $\Phi=6 \times 10^{15} \mathrm{~cm}^{-2}$.

The PI-L spectra consist of four bands: (i) the ultraviolet (UV) spectral band (UV-L) of rather small intensity; (ii) violet (V-L) PI-L peaked at $420 \mathrm{~nm}(2.9$ $\mathrm{eV}$ ); (iii) blue band (B-L) PI-L peaked at around 465 $\mathrm{nm} \quad(\sim 2.7 \mathrm{eV})$; (iv) the yellow-green (YG-L) luminescence band with a peak at $550 \mathrm{~nm}(\sim 2.3 \mathrm{eV})$. Variations of the peak intensity for two the most intensive PI-L bands are depicted in Fig. 4. The broad YG-PI-L emission band suffers a fast luminescence quenching during irradiation. The YG-PI-L band is likely to be attributed to either the $\mathrm{V}_{\mathrm{Ga}} \mathrm{O}_{\mathrm{N}}$ complex [16], [17] or to the carbon-related defects $\mathrm{C}_{\mathrm{N}}$ and $\mathrm{C}_{\mathrm{N}} \mathrm{O}_{\mathrm{N}}$ [17], [18]. The intensity of the B-PL band peaked at $465 \mathrm{~nm}$ decreases with fluence increase. This B-PI-L band might be ascribed to the transitions of carriers within donor-acceptor (D-A) pairs [20]. The intensity of the V-PI-L band increases with irradiation fluence and becomes dominant at the largest fluences of $\Phi>5 \times 10^{14}$ $\mathrm{cm}^{-2}$. This band can emerge due to the formation of vacancies ascribed to radiation defects in the electronblocking AlGaN layer of GaN LEDs [21]. The additional R-PI-L peak at $700 \mathrm{~nm}$ appears in the highly irradiated sensor, which is related to the proton beam excited luminescence in the sapphire substrate, due to $\mathrm{Cr}$ ascribed centres [22].

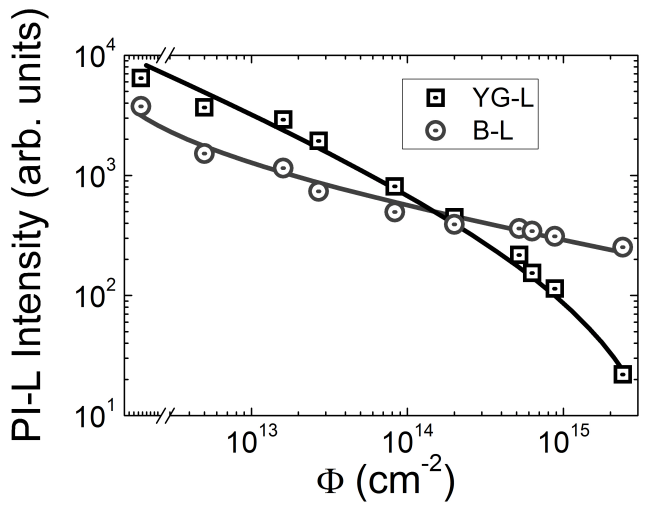

Figure 4. Variations of the PI-L peak-intensities for B and YG spectral bands as a function of proton irradiation fluence

\subsection{CV characteristics and DLTS spectra}

Capacitance-voltage measurements allow for the estimation of the shallow dopant concentration $N_{s}$ and their distribution in the space charge region. Two slopes, typical for the heterostucture-based LED's, can be deduced from the $\mathrm{C}^{-2}-\mathrm{V}$ characteristics (inset for Fig. 5). Each slope can be ascribed to the n- and p-doped layers having different dopant concentrations. The turning-point within C-V's indicates a full-depletion voltage for the less doped layer. The effective doping concentration evaluated from $\mathrm{C}-\mathrm{V}$ characteristics are estimated $N_{\text {eff }}=4 \times 10^{17} \mathrm{~cm}^{-3}$ for the pristine sensor and $N_{\text {eff }}=2.2 \times 10^{17} \mathrm{~cm}^{-3}$ for the sensor irradiated with the highest fluence. These values are in rather good agreement with $N_{\text {eff }}$ values extracted from BELIV measurement. The dopant removal is the main radiation-damage effect in semiconductor radiationdetectors. Variations of capacitance for the heavily irradiated sensor do not exceed $10 \%$ of the average value being of the same magnitude with measurement errors. This result implies a weak dependence of capacitance on reverse voltage in heavily irradiated sensor. It has also been deduced from C-V's (Fig. 5) that values of barrier capacitance in a sensor, irradiated with a proton fluence of $6 \times 10^{15} \mathrm{~cm}^{-2}$ is decreased by a factor of 3.6 , at least, compared to its value in the pristine sensor, due to the decrease of the effective dopant concentration $\left(N_{e f f}=N_{D}-N_{A}\right)$. The $\mathrm{C}^{-2}-\mathrm{V}$ characteristics for the pristine and heavily irradiated samples also indicate rather thin $(d<200 \mathrm{~nm}) \mathrm{n}$ - and pdoped GaN layers.

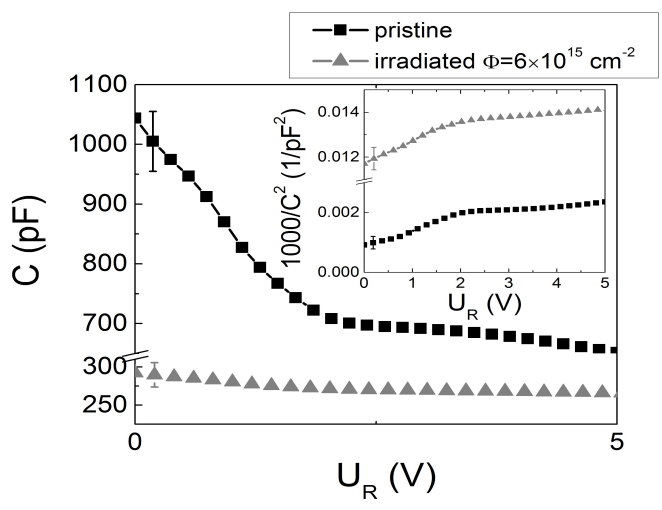

Figure 5. $\mathrm{C}-\mathrm{V}$ characteristics obtained for the pristine $\mathrm{GaN}$ sensor and for that irradiated with the proton fluence of $6 \times 10^{15} \mathrm{~cm}^{-2}$. $\mathrm{C}^{-2}-\mathrm{V}$ plots are depicted in the inset

The distribution of the concentration of shallow centres $N_{s}$ within depth of the space charge regions $x_{d}$ has been evaluated by using $\mathrm{C}-\mathrm{V}$ data. The $N_{s}\left(x_{d}\right)$ is expressed as $N_{s}\left(x_{d}\left(U_{R}\right)\right)=2 / q \varepsilon_{o} \varepsilon S^{2}\left(d\left(1 / C^{2}\right) / d U_{R}\right)$, where $x_{d}\left(U_{R}\right)=\left(2 \varepsilon_{o} \varepsilon U_{R} / q N_{s}\right)^{1 / 2}, q$ is the elementary charge, $\varepsilon_{o}$ is vacuum permittivity, $\varepsilon$ is the material permittivity and $S$ is the area of the heterostructure. Profiling of $N_{s}$ depth distribution was performed using the analysis instruments installed within HERA-DLTS spectrometer. A step-like profile of the $N_{s}$ distribution obtained for the irradiated sensor implies the radiation damage in both layers of the heterostucture. The absolute $N_{s}$ values ascribed to the less-doped layer (for 
D. Meskauskaite et al., Characteristics of 1.6 MeV proton-irradiated..., Rad. Applic., 2017, 2, 2, 118-123

$x_{d} \leq 90 \mathrm{~nm}$ in Fig. 6) correlate well with those $N_{e f f}$ parameters extracted from C-V's and BELIV transients. The $N_{s}$ values in irradiated sensor for $x_{d}<100 \mathrm{~nm}$ are decreased relative to that in the pristine sample and their distribution for both $\mathrm{n}-$ and $\mathrm{p}-\mathrm{GaN}$ layers shows a nearly flat profile. The depth profile of $N_{s}$ (Fig. 6) obtained for the pristine sensor exhibits a sharp peak, which can be related to the MQW at heavydoped layer. However, the $N_{s}$ profiling errors and intricate structure of layers allow only the qualitative tracing of dopant depth-distribution.

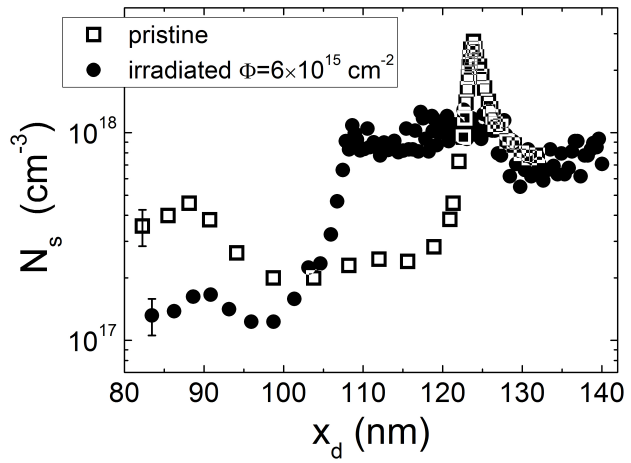

Figure 6. The distribution of the shallow centre concentration $N_{s}$ within sensor depth obtained for the pristine sensor and the sensor irradiated with $6 \times 10^{15} \mathrm{~cm}^{-2}$ fluence

The C-DLTS spectra recorded for the pristine and the irradiated sensor are illustrated in Fig. 7. These DLTS measurements were performed over a temperature range of $10-400 \mathrm{~K}$ using filling pulse of 1.5 $\mathrm{V}$ voltage. To insure the complete filling of traps, the $500 \mathrm{~ms}$ injection pulses were employed. The reverse bias voltage was varied in the range from $1 \mathrm{~V}$ to $10 \mathrm{~V}$, which corresponds to the scan depths of about $80-140$ $\mathrm{nm}$. Rate windows were varied from $1 \mathrm{~ms}$ to $1 \mathrm{~s}$.

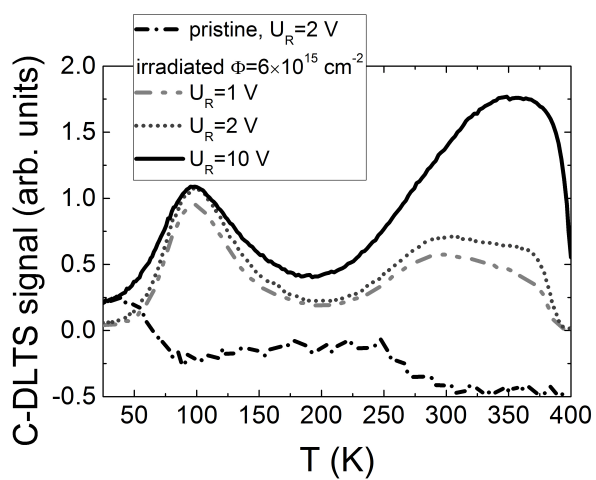

Figure 7. C-DLTS spectra recorded on the pristine sensor and sensor irradiated with proton fluence of $6 \times 10^{15} \mathrm{~cm}^{-2}$

The post-irradiation DLTS spectroscopy on sensors was performed by comparing these spectra with that obtained before irradiations. The DLTS signals on the noise level have only been detected for the pristine sensor (Fig. 7). While, a spectral structure containing broad peaks has been observed for the irradiated sensors. The radiation defect concentration varied near-linearly with irradiation fluence for the examined $\Phi$ range. It has been obtained that the amplitude of a spectral peak $\left(E_{T 1}\right)$ at $100 \mathrm{~K}$ is independent of the reverse-bias voltage $U_{R}$. This result implies a nearlyhomogeneous distribution of the radiation-defect concentration within the depletion region. The broad DLTS spectral band, covering the temperature range of 250-350 K, clearly indicates the overlapping of two spectral peaks, at least.

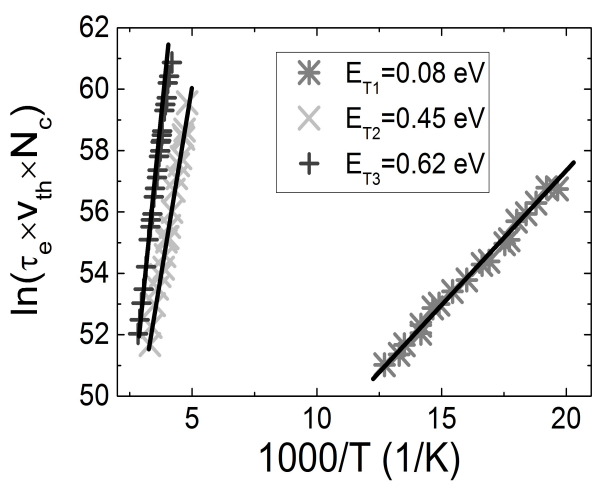

Figure 8. The Arrhenius plots obtained for several spectral peaks recorded on the sensor irradiated with proton fluence of $6 \times 10^{15} \mathrm{~cm}^{-2}$

The amplitude of the latter $\left(E_{T 2}, E_{T 3}\right)$ peaks increases with enhancement of the reverse-bias voltage. This points to a non-uniform distribution of concentration of the radiation-defects $\left(E_{T_{2}}, E_{T_{3}}\right)$ within the deeper regions of heterostructure materials, far from the interface layer. The Arrhenius plots have been displayed by using PhysTech software. The Arrhenius plots (Fig. 8) have been depicted using the relevant shifts of the peak positions over the temperature scale, dependent on the rate-window and on the correlation function (available within a PhysTech software).

Values of the thermal-activation energy $E_{T}$, of trap concentration $N_{T}$ and of their capture cross-section $\sigma$, extracted from analysis of the Arrhenius plots, are listed in Table 1, as estimated for several deep traps.

Table 1. Parameters of traps for sensor irradiated with a proton fluence of $6 \times 10^{15} \mathrm{~cm}^{-2}$ evaluated using Arrhenius plots

\begin{tabular}{|c|c|c|c|}
\hline Level & $E_{C}-E_{T}(\mathrm{eV})$ & $N_{T}\left(\mathrm{~cm}^{-3}\right)$ & $\sigma\left(\mathrm{cm}^{2}\right)$ \\
\hline $\mathrm{E}_{\mathrm{T} 1}$ & 0.08 & $2.6 \times 10^{15}$ & $2 \times 10^{-18}$ \\
$\mathrm{E}_{\mathrm{T} 2}$ & 0.45 & $2 \times 10^{15}$ & $6 \times 10^{-16}$ \\
$\mathrm{E}_{\mathrm{T} 3}$ & 0.62 & $1.8 \times 10^{15}$ & $2 \times 10^{-13}$ \\
\hline
\end{tabular}

The traps, characterized by sets of the $\left(E_{T}, N_{T}, \sigma\right)$ parameters have been attributed to the radiationinduced defects, as follows: i) the traps with activation energy of $E_{T_{1}}=0.08 \mathrm{eV}$ are ascribed to the nitrogen vacancy $\mathrm{V}_{\mathrm{N}}$ [23]; ii) the deep levels with $E_{T 2}=0.45 \mathrm{eV}$ are associated with radiation-defect complexes of rather shallow donors [21]-[22]; iii) the centres characterized by the $E_{T_{3}}=0.62 \mathrm{eV}$ are attributed to the unidentified defect, which has been observed by several authors [23]-[24]. 
D. Meskauskaite et al., Characteristics of $1.6 \mathrm{MeV}$ proton-irradiated ..., Rad. Applic., 2017, 2, 2, 118-123

\section{CONCLUSIONS}

The sensors based on $\mathrm{InGaN} / \mathrm{GaN}$ heterostructures have been studied in order to evaluate the radiation tolerance of such types of detectors and to trace the evolution of the dominant radiation defects with enhancement of irradiation fluence. The pristine heterostructure sensors, as deduced from the temperature-dependent BELIV transients, contain low concentration of grown-in defects. Examination of the in situ evolution of proton-beam excited luminescence (PI-L) and BELIV transients, during 1.6 MeV proton irradiation, allowed to identify the full-depletion state for the heavily irradiated sensors and to separate the channels of the radiative recombination.

Scanning of the effective doping $N_{s}$ depthdistribution within InGaN/GaN heterostructure showed $N_{s}(x)$ profile of step-like shape with rather flat segments, attributed to both $\mathrm{n}$ - and $\mathrm{p}-\mathrm{GaN}$ layers, for the sensors irradiated with proton fluence of $6 \times 10^{15}$ $\mathrm{cm}^{-2}$.

The radiation defects, ascribed to the nitrogen vacancy, to the unidentified defect, and to the complexes of shallow donors have been revealed by using C-DLTS technique.

The potential for the application of GaN-based sensors by detecting both the electrical and optical signals in a harsh radiation environment has also been demonstrated in this study. Detection of both the electrical and optical signals also may be applicable for characterization of HEMTs [27]-[29].

Acknowledgement: This research was partially supported by the Research Council of Lithuania, grant LAT-01/2016. DM thanks the Research Council of Lithuania for financial support in RAD 2017 Conference attendance.

\section{REFERENCES}

1. S. Fujita, "Wide-bandgap semiconductor materials: For their full bloom," Jpn. J. Appl. Phys., vol. 54, no. 3, p. 30101, Feb. 2015. DOI: 10.7567/JJAP.54.030101

2. S. J. Pearton et al., "GaN-based diodes and transistors for chemical, gas, biological and pressure sensing," $J$. Phys. Condens. Matter, vol. 16, no. 29, pp. R961-R994, Jul. 2004. DOI: $10.1088 / 0953-8984 / 16 / 29 /$ Ro2

3. M. Sugiura et al., "Study of radiation detection properties of GaN pn diode," Jpn. J. Appl. Phys., vol. 55, no. 5S, p. 05FJo2, Mar. 2016. DOI: $10.7567 / J J A P .55 .05 F J 02$

4. M. Moll, "Radiation tolerant semiconductor sensors for tracking detectors," Nucl. Instruments Methods Phys. Res. Sect. A Accel. Spectrometers, Detect. Assoc. Equip., vol. 565, no. 1, pp. 202-211, Sep. 2006. DOI: 10.1016/j.nima.2006.05.001

5. P. J. Sellin and J. Vaitkus, "New materials for radiation hard semiconductor dectectors," Nucl. Instrum. Methods Phys. Res. Sect. A-Accel. Spectrom. Dect. Assoc. Equip., vol. 557, no. 2, pp. 479-489, Feb. 2006. DOI: 10.1016/j.nima.2005.10.128

6. E. Gaubas et al., "Correlative analysis of the in situ changes of carrier decay and proton induced photoluminescence characteristics in chemical vapor deposition grown GaN," Appl. Phys. Lett., vol. 104, no. 6, p. 62104, Jan. 2014.

DOI: $10.1063 / 1.4865499$

7. P. Pittet et al., "PL characterization of $\mathrm{GaN}$ scintillator for radioluminescence-based dosimetry," Opt. Mater. (Amst)., vol. 31, no. 10, pp. 1421-1424, Aug. 2009.

DOI: 10.1016/j.optmat.2008.09.012

8. Xeon 1 Power Blue LED OSB4XNE1E1E VER C.3, OptoSupply International, Hong Kong, China.

Retrieved from:

http://www.optosupply.com/uppic/201686103526.pdf Retrieved on: Apr. 26, 2017

9. E. Gaubas, T. Ceponis and J.V. Vaitkus, Pulsed capacitance technique for evaluation of barrier structures. Saarbrucken-Berlin: LAMBERT Academic Publishing, 2013.

10. J. F. Ziegler, M. D. Ziegler and J. P. Biersack, "SRIM The stopping and range of ions in matter (2010)," Nucl. Instruments Methods Phys. Res. Sect. B Beam Interact. with Mater. Atoms, vol. 268, no. 11-12, pp. 1818-1823, Jun. 2010.

DOI: 10.1016/j.nimb.2010.02.091

11. E. Gaubas, A. Uleckas, J. Vaitkus, J. Raisanen and P. Tikkanen, "Instrumentation for the in situ control of carrier recombination characteristics during irradiation by protons," Rev. Sci. Instrum., vol. 81, no. 5, p. 53303 , May 2010.

DOI: $10.1063 / 1.3429944$

PMid: 20515132

12. D. V. Lang, "Deep-level transient spectroscopy: A new method to characterize traps in semiconductors," J. Appl. Phys., vol. 45, no. 7, pp. 3023-3032, 1974. DOI: $10.1063 / 1.1663719$

13. FT 1030 Deep-Level Transient Spectroscopy System, PhysTech GmbH, Moosburg, Germany.

Retrieved from:

http://www.phystech.de/products/dlts/dlts.htm

Retrieved on: Apr. 26, 2017

14. E. Gaubas et al., "Correlated evolution of barrier capacitance charging, generation, and drift currents and of carrier lifetime in Si structures during $25 \mathrm{MeV}$ neutrons irradiation," Appl. Phys. Lett., vol. 101, no. 23, p. 232104, Dec. 2012.

DOI: $10.1063 / 1.4769370$

15. E. Gaubas, I. Brytavskyi, T. Ceponis, V. Kalendra and A. Tekorius, "Spectroscopy of deep traps in $\mathrm{Cu}_{2} \mathrm{~S}-\mathrm{CdS}$ junction structures," Materials, vol. 5, no. 12, pp. 25972608, Dec. 2012.

DOI: $10.3390 / \mathrm{ma} 5122597$

16. M. A. Reshchikov, H. Morkoc, S. S. Park and K. Y. Lee, "Two charge states of dominant acceptor in unintentionally doped GaN: Evidence from photoluminescence study," Appl. Phys. Lett., vol. 81, no. 26, p. 4970, Dec. 2002. DOI: $10.1063 / 1.1531227$

17. J. Rodrigues et al., "Influence of neutron irradiation and annealing on the optical properties of GaN," Phys. Status Solidi $C$, vol. 9, no. 3-4, pp. 1016-1020, Feb. 2012.

DOI: $10.1002 /$ pssc. 201100200

18. M. A. Reshchikov, D. O. Demchenko, A. Usikov, H. Helava and Y. Makarov, "Carbon defects as sources of the green and yellow luminescence bands in undoped GaN," Phys. Rev. B, vol. 90, no. 23, p. 235203, Dec. 2014.

DOI: 10.1103/PhysRevB.90.235203

19. J. L. Lyons, A. Janotti and C. G. Van de Walle, "Carbon impurities and the yellow luminescence in GaN," Appl. Phys. Lett., vol. 97, no. 15, p. 152108, Oct. 2010. DOI: $10.1063 / 1.3492841$

20. M. A. Reshchikov and H. Morkoc, "Luminescence properties of defects in GaN," J. Appl. Phys., vol. 97, no. 6, p. 61301, Mar. 2005. 
D. Meskauskaite et al., Characteristics of 1.6 MeV proton-irradiated..., Rad. Applic., 2017, 2, 2, 118-123

DOI: $10.1063 / 1.1868059$

21. N. Nepal, M. L. Nakarmi, J.Y. Lin and H.X. Jiang, "Photoluminescence studies of impurity transitions in AlGaN alloys," Appl. Phys. Lett., vol. 89, no. 9, p. 92107, Aug. 2006. DOI: $10.1063 / 1.2337856$

22. K. H. Lee and J. H. Crawford, "Luminescence of the F center in sapphire," Phys. Rev. B, vol. 19, no. 6, pp. 3217-3221, Mar. 1979 .

DOI: 10.1103/PhysRevB.19.3217

23. A. Castaldini, A. Cavallini, A. Castaldini and L. Polenta, "Deep levels and irradiation effects in n-GaN," J. Phys Condens. Matter, vol. 12, no. 49, pp. 10161-10167, 2000.

DOI: $10.1088 / 0953-8984 / 12 / 49 / 315$

24. A. Y. Polyakov et al., "Radiation effects in GaN materials and devices," J. Mater. Chem. C, vol. 1, no. 5, pp. $877-887,2013$ DOI: $10.1039 / \mathrm{C} 2 \mathrm{TCooo} 39 \mathrm{C}$

25. S. J. Pearton, R. Deist, F. Ren, L. Liu, A. Y. Polyakov and J. Kim, "Review of radiation damage in GaN-based materials and devices," J. Vac. Sci. Technol. A, vol. 31 no. 5, p. 50801, Apr. 2013.

DOI: $10.1116 / 1.4799504$

26. A. Hierro et al., "Optically and thermally detected deep levels in n -type Schottky and p+-n GaN diodes," Appl. Phys. Lett., vol. 76, no. 21, pp. 3064-3066, May 2000. DOI: $10.1063 / 1.126580$

27. E. Patrick et al., "Modeling proton irradiation in $\mathrm{AlGaN} / \mathrm{GaN}$ HEMTs: understanding the increase of critical voltage," IEEE Trans. Nucl. Sci., vol. 60, no. 6, pp. 4103-4108, Dec. 2013. DOI: $10.1109 /$ TNS.2013.2286115

28. B. D. Weaver et al., "On the radiation tolerance of AlGaN/GaN HEMTs," ECS J. Solid State Sci. Technol., vol. 5, no. 7, pp. Q208-Q212, Jun. 2016. DOI: $10.1149 / 2.0281607$ jss

29. E. E. Patrick, M. Choudhury, F. Ren, S. J. Pearton and M. E. Law, "Simulation of radiation effects in AlGaN/GaN HEMTs," ECS J. Solid State Sci. Technol., vol. 4, no. 3, pp. Q21-Q25, Jan. 2015.

DOI: $10.1149 / 2.0181503 j s s$ 